Cahiers « Mondes anciens »

ANCIENS

Histoire et anthropologie des mondes anciens

4 | 2013

Journées doctorales ANHIMA 2010 et 2011

\title{
La crosse et la cruche
}

Symboles de légitimité de l'imperium ou symboles de l'augurat?

Yann Berthelet

\section{(2) OpenEdition}

Journals

Édition électronique

URL : http://journals.openedition.org/mondesanciens/1037

DOI : 10.4000/mondesanciens. 1037

ISSN : 2107-0199

Éditeur

UMR 8210 Anthropologie et Histoire des Mondes Antiques

Référence électronique

Yann Berthelet, « La crosse et la cruche », Cahiers « Mondes anciens » [En ligne], 4 | 2013, mis en ligne le 01 juillet 2013, consulté le 30 avril 2019. URL : http://journals.openedition.org/mondesanciens/1037 ; DOI : 10.4000/mondesanciens. 1037

Ce document a été généré automatiquement le 30 avril 2019

\section{(c) $(1)(9$}

Les Cahiers «Mondes Anciens » sont mis à disposition selon les termes de la licence Creative Commons Attribution - Pas d'Utilisation Commerciale - Pas de Modification 4.0 International. 


\section{La crosse et la cruche}

Symboles de légitimité de l'imperium ou symboles de l'augurat?

\section{Yann Berthelet}

Les instruments cultuels représentés sur les monnaies sont traditionnellement interprétés comme le symbole du sacerdoce auquel appartenait le magistrat responsable de l'émission. Ainsi, de même que l'on voit dans la représentation monétaire d'un trépied l'indice que le magistrat responsable de l'émission était un vir sacris faciundis (RRC 511/1, CRAWFORD 1974, I, p. 520 ; II, pl. LXI ; voir ci-dessous fig. 9), on considère généralement que la présence d'une crosse augurale (lituus) sur la monnaie signifie qu'il était augure ( $\mathrm{T}$ AYLOR 1944 ; FRIER 1967 et 1969 ; FEARS 1975) : ce type d'argument est fréquemment utilisé par les numismates et les prosopographes et constitue même, parfois, la seule preuve de l'augurat (exemple de Quintus Cassius Longinus, $R R C$ 428/3, CRAWFORD 1974, I, p. 452 et II, pl. LII ; RÜPKE 2005, II, n 1105, p. 862, n. 3)1. 
Fig. 1 : Sylla, aureus, 84-83 av. J.-C.

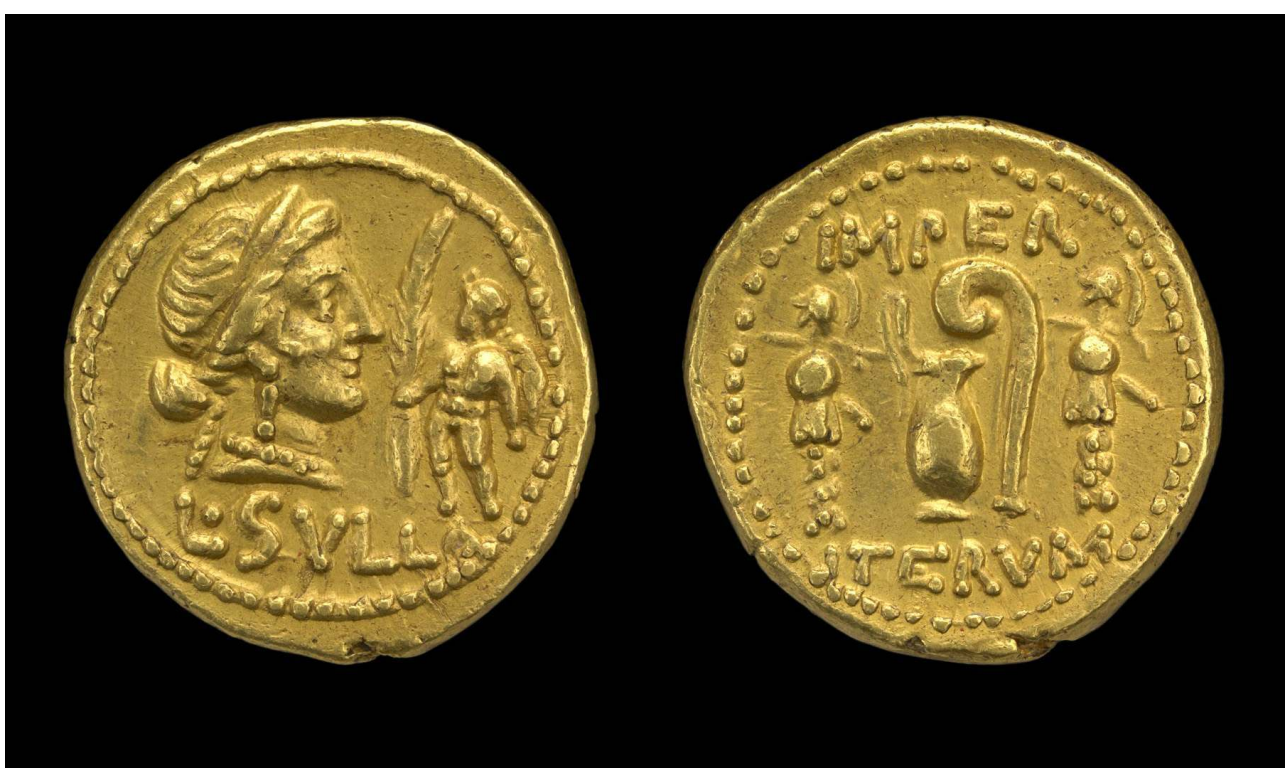

RRC 359/1

(C) The Trustees of the British Museum

2 Cette interprétation traditionnelle a été remise en cause, cependant, pour les monnaies tardo-républicaines. Prenant acte à la fois de la datation en $84-83^{2}$ de l'émission monétaire syllanienne RRC 359/1-2 associant sur le revers une crosse augurale à une cruche (CRAWFORD 1974, I, p. 373-374 et II, pl. XLVII ; voir fig. 1) et de la datation proposée par E. BADIAN (1968), en 82, pour l'obtention de l'augurat par Sylla, plusieurs chercheurs ont considéré que la crosse augurale ne pouvait plus systématiquement être interprétée, à compter de cette émission, comme la preuve d'une appartenance du magistrat responsable de l'émission au collège des augures, mais devait plutôt être comprise comme une référence à la loi curiate et au droit d'auspices qui légitimaient son imperium (C RAWFORD 1974, I, p. 373-374 ; SCHEID 1981, p. 170 ; KEAVENEY 1982, p. 154-161 ; NORTH 1990, p. 533, n. 14 ; LINDERSKI 1996, p. 179-181). R. STEWART (1997), encore suivie récemment par P. ASSENMAKER (à paraître dans RN, 2013; idem, sous presse), a donné un poids supplémentaire à cette nouvelle interprétation, en proposant une explication séduisante de la cruche associée au lituus sur les monnaies de plusieurs détenteurs d'imperium de la fin de la République.

Cette cruche, que R. Stewart désigne du terme jug sans autre précision, semble être un urceus ou un gut $(t) u s$. Ces appellations sont des conventions d'archéologues et d'historiens de l'art appliquées à deux types de vase au col plus resserré que le ventre, difficiles à distinguer l'un de l'autre sur les monnaies. L'urceus, au col plus large que celui du gut $(t) u s$, pouvait servir au lavage des mains du sacrifiant et contenir de l'huile ou d'autres liquides ; il était muni ou non d'anses. Le gut $(t) u s$, au col plus étroit, servait aux libations de vin ou d'huile, et possédait une anse ${ }^{3}$.

4 Rapprochant les cruches représentées sur les monnaies de celles que l'on peut voir sur de nombreux bas-reliefs et qui sont fréquemment associées à des scènes sacrificielles ( $\mathrm{S}$ TEWART 1997, p. 171-173), R. Stewart les interprète non plus en référence au collège augural, comme il était habituel jusque là, mais en référence au collège pontifical, chargé 
de contrôler les sacra ${ }^{4}$. Elle argue alors du rôle des Vestales dans la fabrication de la mola salsa sacrificielle, de l'intervention du Flamen Dialis dans les Vinalia, et du contrôle exercé par les pontifes sur les vignes publiques, pour étayer son interprétation (STEWART 1997, p. 173-174). Constatant que la cruche n'est pas associée au lituus avant l'émission monétaire de Sylla $R R C$ 359/1-2, R. Stewart interprète la frappe conjointe de ces deux symboles sacerdotaux comme une pratique propre aux chefs militaires de la fin de la République, en particulier lorsque, dans un contexte de guerre civile, ils étaient en recherche de légitimité. Alors que le lituus ferait référence à la légitimation, par le collège augural et la loi curiate, des auspices du magistrat, la cruche ferait allusion aux sacrifices accomplis par les consuls lors de leur entrée en charge. En manque de légitimité, ou associés à des hommes en manque de légitimité, les magistrats du $\mathrm{I}^{\mathrm{er}}$ siècle responsables de ces émissions auraient fait frapper les symboles de la cruche et du lituus en une claire référence aux rituels traditionnels de légitimation des pouvoirs du magistrat républicain (STEWART 1997, p. 174-186).

Fig. 2 : Antoine et Lépide, denier, 43-42 av. J.-C.
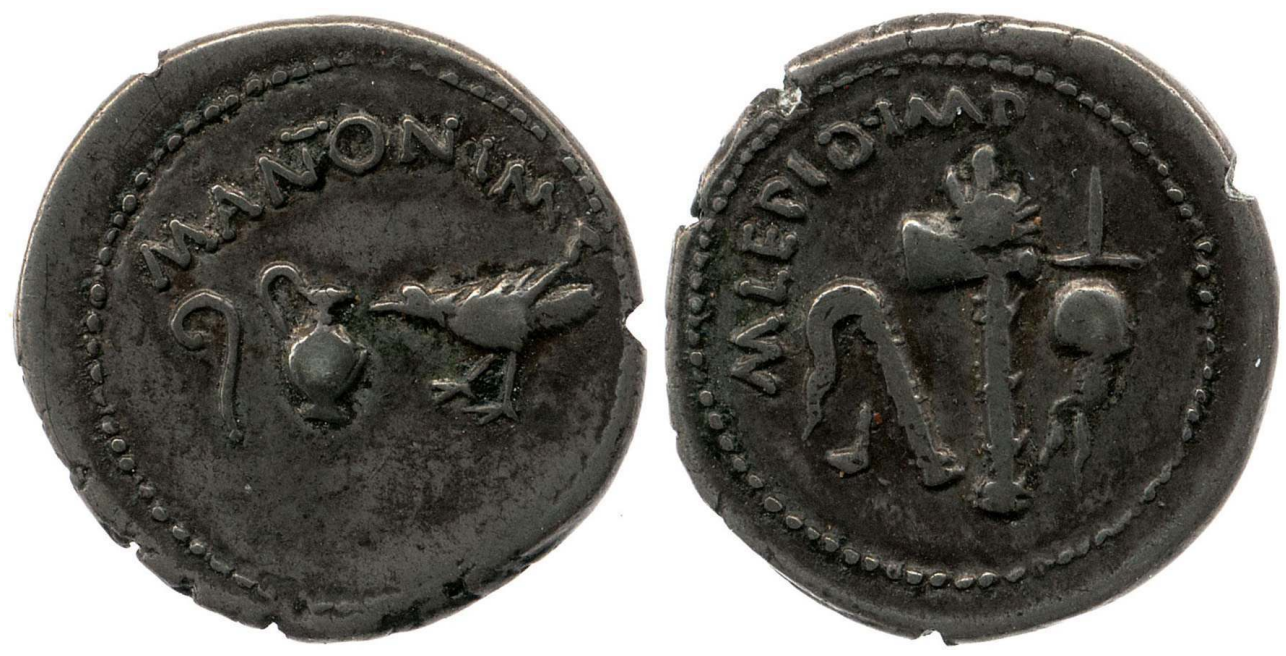

RRC 489/2

(C) THE TRUSTEES OF THE BRITISH MUSEUM

5 À l'examen du dossier, il apparait cependant qu'une émission réalisée par Antoine et Lépide, RRC 489/1-3 (CRAWFORD 1974, I, p. 498-499 et II, pl. LVIII ; voir fig. 2), s'oppose à une telle interprétation "pontificale » de l'urceus/gut(t)us associé au lituus. L'émission est datable entre mai 43, lorsque Antoine, proconsul en Gaule cisalpine et en Gaule chevelue, et Lépide, proconsul en Gaule transalpine et en Espagne citérieure, unirent leurs forces, et le début de l'année 42. Le droit de la monnaie porte le nom et la titulature d'Antoine avec, en dessous, un lituus, un urceus/gut(t)us et un oiseau augural, identifié par M. H. Crawford à un corbeau. Le revers porte le nom et la titulature de Lépide avec, en-dessous, un objet à puiser en forme d'écuelle, du type simpuvium/simpulum, un aspersoir, une hache et un bonnet de flamine. Chaque face est très explicitement associée au personnage nommé par la légende, de sorte qu'il n'est pas possible d'interpréter les symboles sacerdotaux autrement qu'en lien avec chacun des personnages. Or, à cette date, Antoine est augure, de sorte que l'urceus/gut $(t)$ us présent sur le droit consacré à Antoine et absent du revers consacré au grand pontife Lépide, doit être interprété en référence à son augurat. Un autre type de la même émission, RRC 489/4 (CRAWFoRD 1974, I, p. 499 et II, pl. LVIII), 
auquel Lépide n'est pas associé, regroupe les trois mêmes symboles auguraux, le lituus, l' urceus/gut $(t)$ us et l'oiseau.

Fig. 3 : César, aureus, 47-46 av. J.-C.
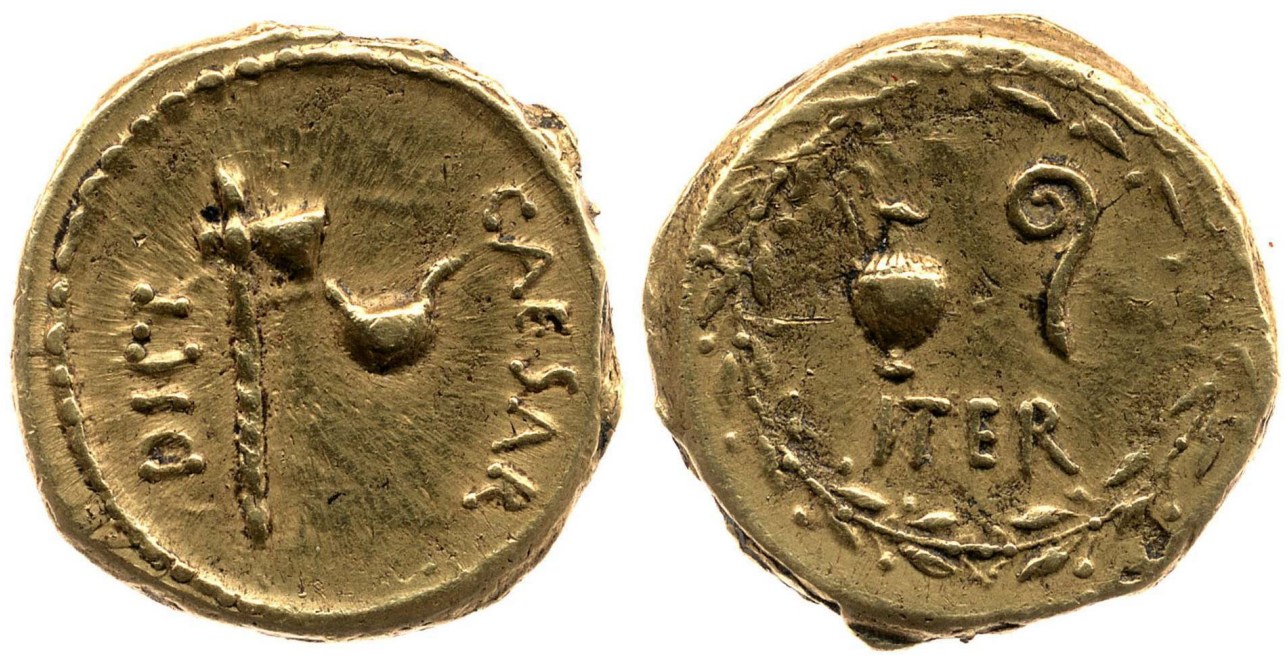

RRC 456/1 A

(C) THE TRUSTEES OF THE BRITISH MUSEUM

De la même manière, une monnaie frappée par César en 47 ou au début 46 ( $R R C 456 / 1$ a et b, CRAWFORD 1974, I, p. 471 et II, pl. LIV ; voir fig. 3), lors de son entrée dans le collège augural (RÜPKE 2005, II, n² 2003, p. 1058), distingue clairement entre les instruments pontificaux, sur le droit, qui renvoient au grand pontificat qu'il occupe depuis 63, et les instruments auguraux, sur le revers, à savoir l'urceus/gut $(t)$ us et le lituus, qui renvoient à sa nouvelle dignité d'augure.

Fig. 4 : Antoine, denier, 43 av. J.-C.
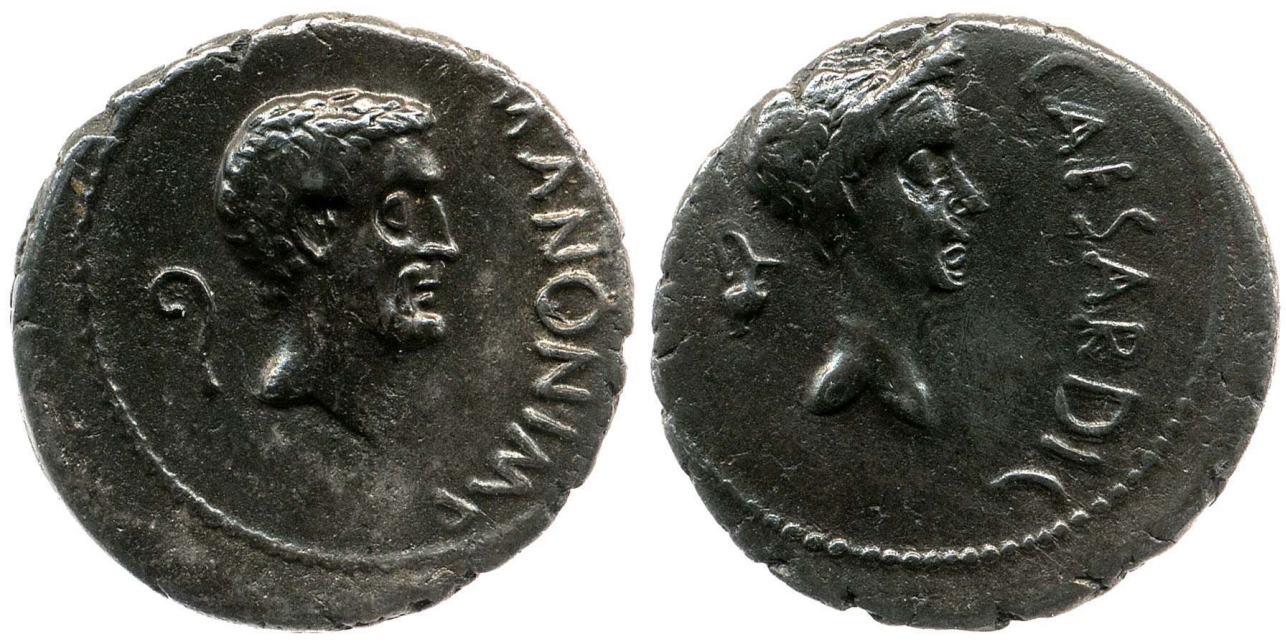

RRC 488/1

(C) THE TRUSTEES OF THE BRITISH MUSEUM

7 L'émission RRC 488/1-2 (CRAWFORD 1974, I, p. 498 et II, pl. LVIII ; voir fig. 4), de 43, qui associe Antoine au lituus sur le droit et César à l'urceus/gut(t)us sur le revers, ne constitue pas une objection valable, puisque César fut non seulement grand pontife, mais aussi 
augure. La cruche associée à César doit donc être comprise comme une référence à son augurat, d'autant plus que c'est Antoine qui émet la monnaie: on peut donc supposer qu'il tient à souligner que César, dont il se proclame l'héritier, appartenait au même collège sacerdotal que lui.

Fig. 5 : Antoine et Octavien, denier, 41 av. J.-C.
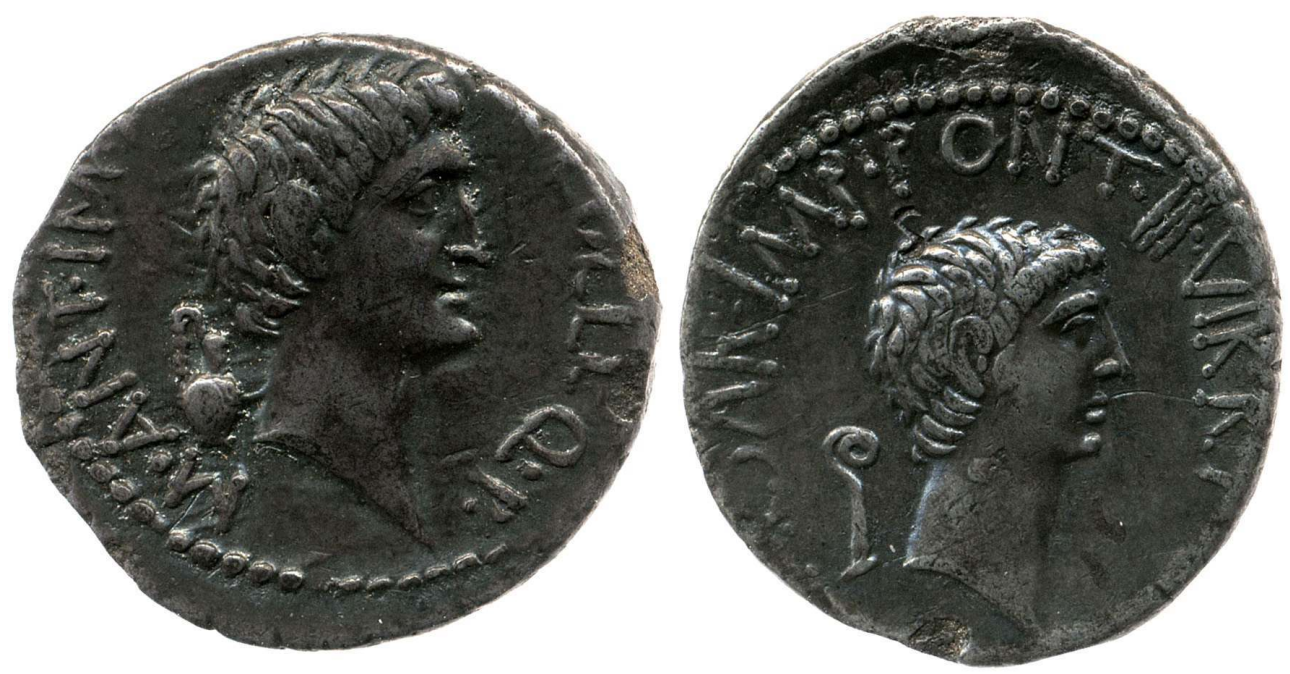

RRC 517/8

(C) THE TRUSTEES OF THE BRITISH MUSEUM

8 En outre, une pièce qu'il émit en 41 en commun avec Octavien, RRC 517/7-8 (CRAWFoRD 1974, I, p. 526 et II, pl. LXII ; voir ci-dessous fig. 5), associe cette fois l'urceus/gut(t)us à Antoine, qualifié d'augure, sur le droit, et le lituus au jeune César, certes qualifié de pontife, mais qui était également augure depuis 43 (RÜPKE 2005, II, n 1012, p. 839 et n. 3), sur le revers. Enfin, on ne trouve jamais, sur les monnaies, d'urceus/gut $(t) u s$ associé à la fonction pontificale (DRUMmond 2008, p. 392), mais des simpuvia/simpula comme sur le revers de RRC 489/1-3 (CRAWFORD 1974, I, p. 498-499 et II, pl. LVIII ; voir ci-dessus fig. 2) et de RRC 532/1 (CRAWFORD 1974, I, p. 533-534 et II, pl. LXIII) ou des vases du type culullus comme sur le revers de $R R C$ 406/1 (CRAWFORD 1974, I, p. 418 et II, pl. L), sur le droit de RRC 456/1 a et b (CRAWFORD 1974, I, p. 471 et II, pl. LIV; voir ci-dessus fig. 3) ou sur le revers de RRC 484/1 (CRAWFORD 1974, I, p. 496 et II, pl. LVII) et de RRC 508/1-2 (CRAWFORD 1974, I, p. 518 et II, pl. LXI). 
Fig. 6 : César, denier, 44 av. J.-C.
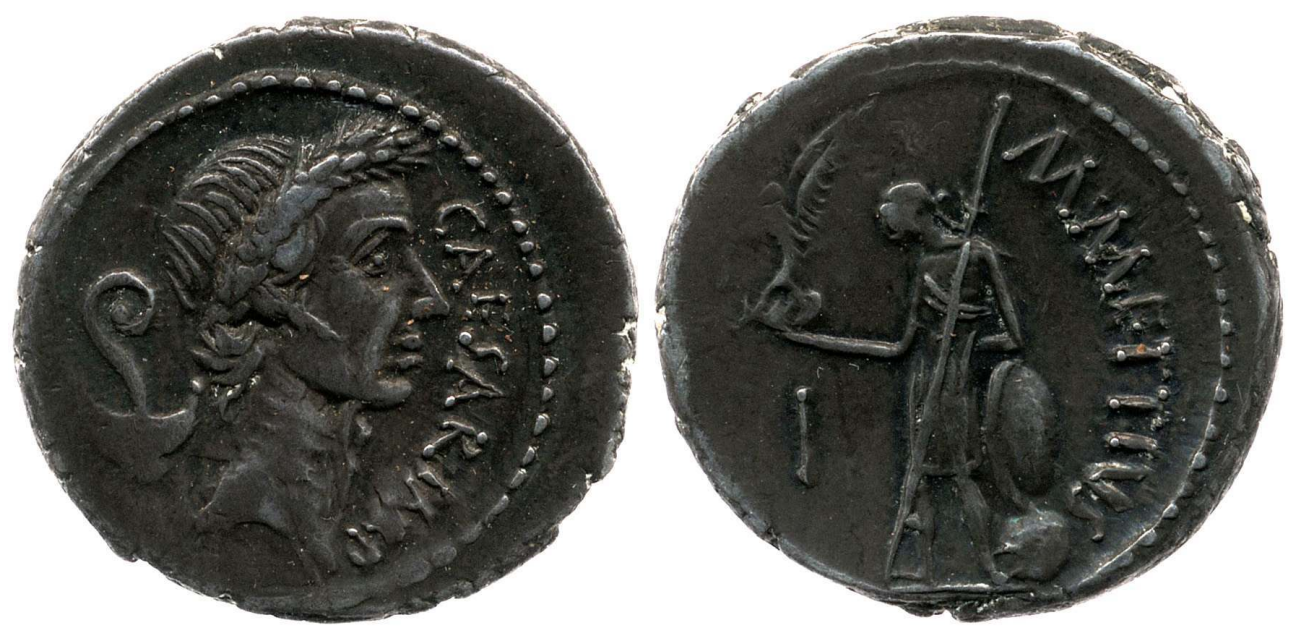

RRC 480/3

(C) THE TRUSTEES OF THE BRITISH MUSEUM

9 Les archéologues et les historiens de l'art appellent simpuvium/simpulum un petit objet à puiser pourvu d'un long manche, dont la forme est proche de celle d'une écuelle, et culullus un vase largement ouvert, sans pied ou avec seulement un petit pied, destiné à recevoir le sang des victimes ${ }^{5}$. Ces derniers ne semblent associés au lituus que sur les monnaies de César (lituus et culullus: RRC 480/3, CRAWFORD 1974, I, p. 488 et II, pl. LVI ; voir fig. 6) ou d'Auguste et de ses successeurs (lituus et simpuvium/simpulum : à titre d'exemple, pour Auguste, RIC 402, CARSON et SUtherLAND, 1984, I, p. 72 ; pour Caligula, RIC 63, ibidem, p.113), c'est-à-dire de personnalités qui cumulèrent, contrairement à la tradition républicaine ${ }^{6}$, l'augurat et le pontificat, et même le grand pontificat.

Or une brève enquête prosopographique suffit à prouver que les émissions monétaires tardo-républicaines qui associent l'urceus/gut(t)us au lituus sont, à trois exceptions près, le fait d'augures :

- en 71, Pompée (RRC 402/1 a et b, CRAWFORD 1974, I, p. 83 et 412-413 ; II, pl. L ; RÜPKE 2005, II, n - 2756, p. 1219, n. 2).

- en 56, Faustus Cornelius Sulla Felix (RRC 426/3, CRAWFORD 1974, I, p. 449 ; II, pl. LI), attesté comme augure dès 57 (RÜPKE 2005, II, $n^{\circ}$ 1388, p. 926).

- en 47 ou au début 46, lors de son admission au sein du collège augural, César ( $R R C 456 / 1$ a et b, CRAWFORD 1974, I, p. 471 ; II, pl. LIV ; cf. RÜPKE 2005, II, n 2003, p. 1058).

- en 43-42, Publius Cornelius Lentulus Spinther (RRC 500/1-7, CRAWFORD 1974, I, p. 514 ; II, pl. LXI), augure depuis 57 (RÜPKE 2005, II, n 1354, p. 918).

- en 39, Antoine (RRC 522/1-4, CRAWFORD 1974, I, p. 528 ; II, pl. LXIII), augure depuis 50 (RÜPKE 2005, II, n669, p. 770). 
Fig. 7 : Quintus Caecilius Metellus Pius, denier, 81 av. J.-C.
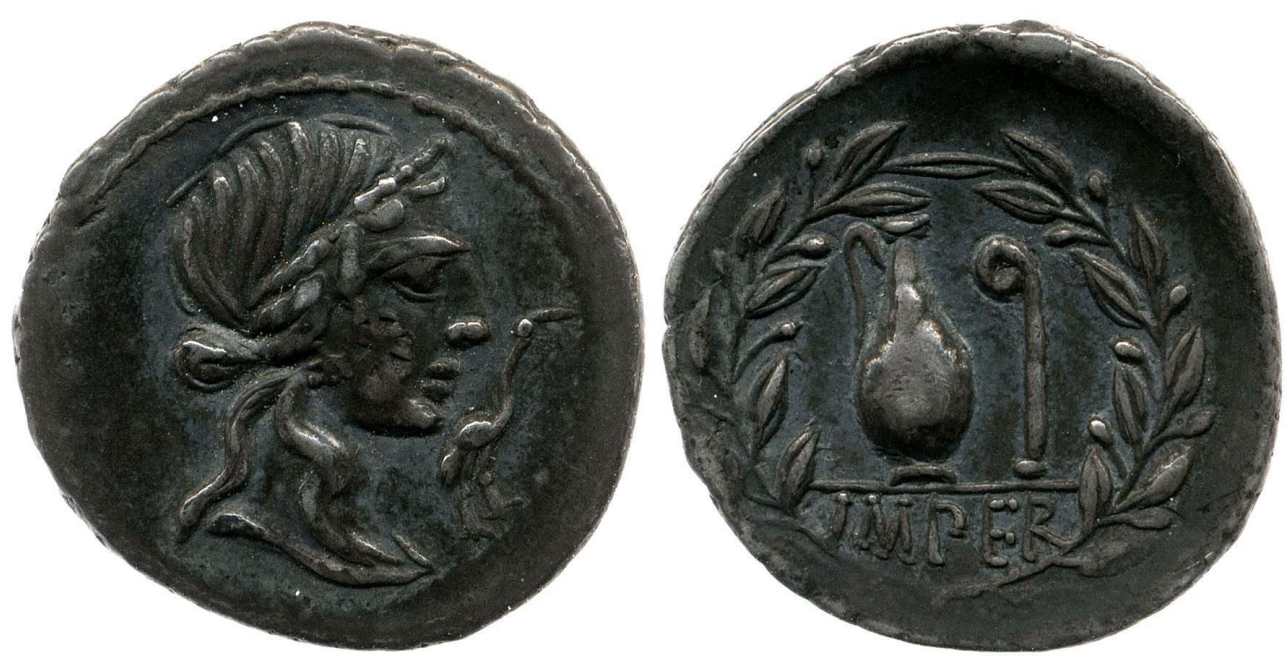

RRC 374/2

(C) THE TRUSTEES OF THE BRITISH MUSEUM

Fig. 8 : Quintus Caecilius Metellus Pius Scipion Nasica, denier, 47-46, av. J.-C.
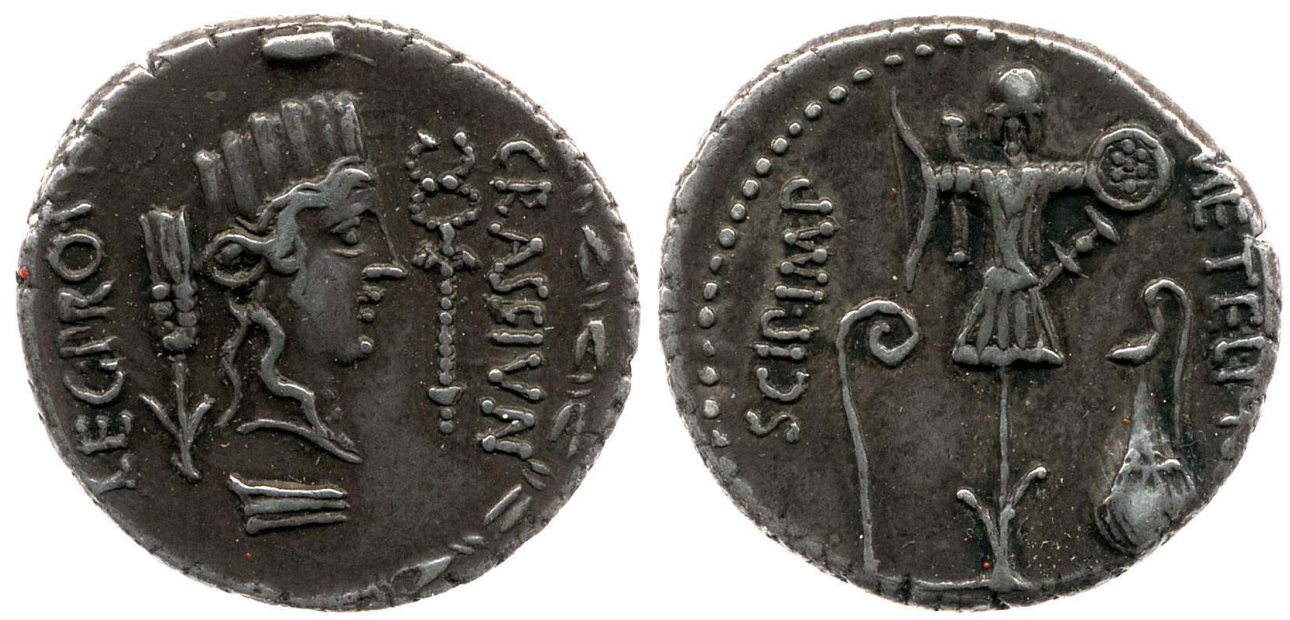

RRC 460/3

(C) THE TRUSTEES OF THE BRITISH MUSEUM

11 Seules font exception les émissions de Quintus Caecilius Metellus Pius, en 81 (RRC 374/2, C RAWFORD 1974, I, p. 390 ; II, pl. XLVIII ; voir fig. 7), de Quintus Caecilius Metellus Pius Scipio Nasica, en 47-46 (RRC 460/3, CRAWFORD 1974, I, p. 472 ; II, pl. LIV; voir ci-dessous fig. 8) et de Sextus Pompée, en 42-39 (RRC 511/3 a, b et c, CRAWFORD 1974, I, p. 520 ; II, pl. LXII ; ЕsтIOT 2006 ; voir ci-dessous fig. 9 et 10).

12 Quintus Caecilius Metellus Pius et Quintus Caecilius Metellus Pius Scipio Nasica sont attestés comme pontifes, et le premier comme grand pontife (RÜPKE 2005, II, n 985 et n - 986, p. 833), mais jamais comme augures. Le cumul de ces sacerdoces étant difficilement envisageable ${ }^{7}$, il a été proposé de voir les insignes auguraux présents sur leurs émissions monétaires comme une référence à un hypothétique augurat détenu par leur ancêtre Quintus Caecilius Metellus Numidicus (TAYLOR 1944, p. 354 ; LINDERSKI 1996, p. 178-179 ; R ÜPKE 2005, II, n 984, p. 832-833). Si l'hypothèse peut paraître fragile (NORTH 1990, p. 533, 
n. 14), trois arguments la rendent néanmoins plausible : il est tout à fait improbable qu'un personnage de l'importance de Quintus Caecilius Metellus Numidicus, pour lequel aucun sacerdoce ne nous est connu, n'ait pas détenu de prêtrise importante; il existe d'autres émissions monétaires qui relèvent de la piété filiale, comme RRC 434/2 (CRAWFORD 1974, I, p. 456 ; II, pl. LII), où Quintus Pompeius Rufus rappelle l'appartenance de son grand-père au collège des décemvirs sacris faciundis, en lui attribuant la flèche et la branche de laurier d'Apollon; une telle piété, enfin, est précisément mise en avant sur les émissions monétaires de Quintus Caecilius Metellus Pius et de Quintus Caecilius Metellus Pius Scipio Nasica, qui portent tous deux le cognomen «Pius». Sur son émission, Quintus Caecilius Metellus Pius associe les insignes auguraux (de son père, selon notre hypothèse) à la figure de la Pietas ; figure qu'il associe, sur un autre type, aux éléphants puniques capturés en 251 par son ancêtre Lucius Caecilius Metellus (RRC 374/1, CRAWFORD 1974, I, p. 390 ; II, pl. XLVIII). Concernant l'émission de Quintus Caecilius Metellus Pius Scipio Nasica, on notera la présence, sur la face portant les insignes auguraux (de son grand-père par adoption, selon notre hypothèse), juste à côté de l'urceus/gut(t)us, du cognomen "Pius » (METEL. PIVS) : or, il fit frapper cette émission en Afrique, sur les terres mêmes où son aïeul par adoption avait acquis le cognomen de victoire « Numidicus».

Fig. 9 : Sextus Pompée, aureus, 42-39 av. J.-C.

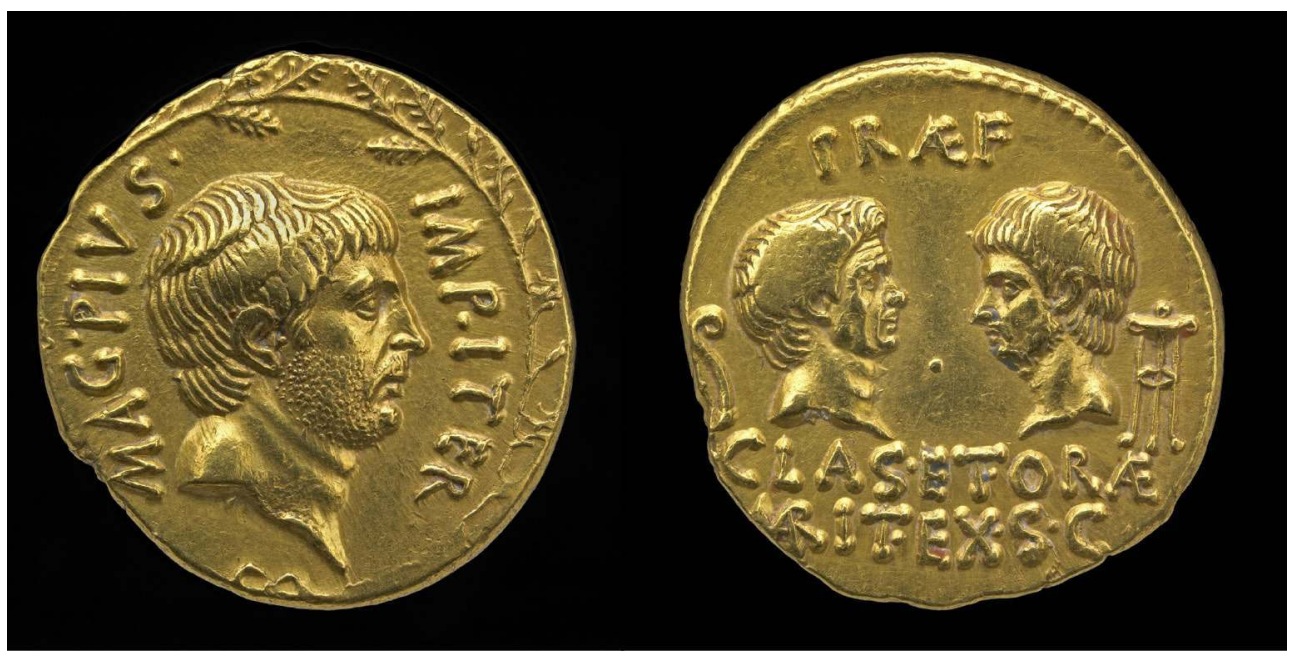

RRC 511/1

(C) THE TRUSTEES OF THE BRITISH MUSEUM

La série monétaire $R R C 511$ émise par Sextus Pompée en Sicile (voir fig. 9 et 10) conforte cette interprétation. Sa datation, reprise récemment par S. Еsтіот (2006) avec des arguments convaincants, doit être établie entre la victoire sur $\mathrm{Q}$. Salvidienus Rufus à l'été 42 et le traité de Misène à l'été 39. Comme le prouvent l'association des symboles auguraux (lituus seul, en $R R C 511 / 1$; lituus et urceus/gut $(t) u s$, en $R R C 511 / 3$ ) à la figure de son père - parallèlement, en $R R C 511 / 1$, à l'association du trépied des quindécimvirs sacris faciundis à la figure de son frère - et l'importance du thème de la Pietas sur les types de l'émission $R R C 511^{8}$, à travers la légende "MAG. PIVS », la cruche et le lituus du type RRC 511/3 renvoient non pas à l'augurat de Sextus Pompée, plus tardif, mais à celui de Pompée le Grand :

L'aureus $R R C$ 511/1, le denier $R R C 511 / 3$ ménagent une place essentielle à l'image du père et $\mathrm{du}$ frère, chaque fois accompagnés des symboles de leurs charges 
sacerdotales : Pompée le Grand avec la cruche et le lituus, ou le lituus seul ; Cnaeus avec le trépied du XVvir sacris faciundis. Fait significatif et essentiel pour nos datations : alors que le droit de l'aureus RRC 511/1 présente l'effigie de Sextus luimême, les attributs sacerdotaux sont en revanche absents, et pour cause : la charge d'augure ne lui sera attribuée que plus tard, en août 39, par le traité de Misène9.

Fig. 10 : Sextus Pompée, denier, 42-39 av. J.-C.
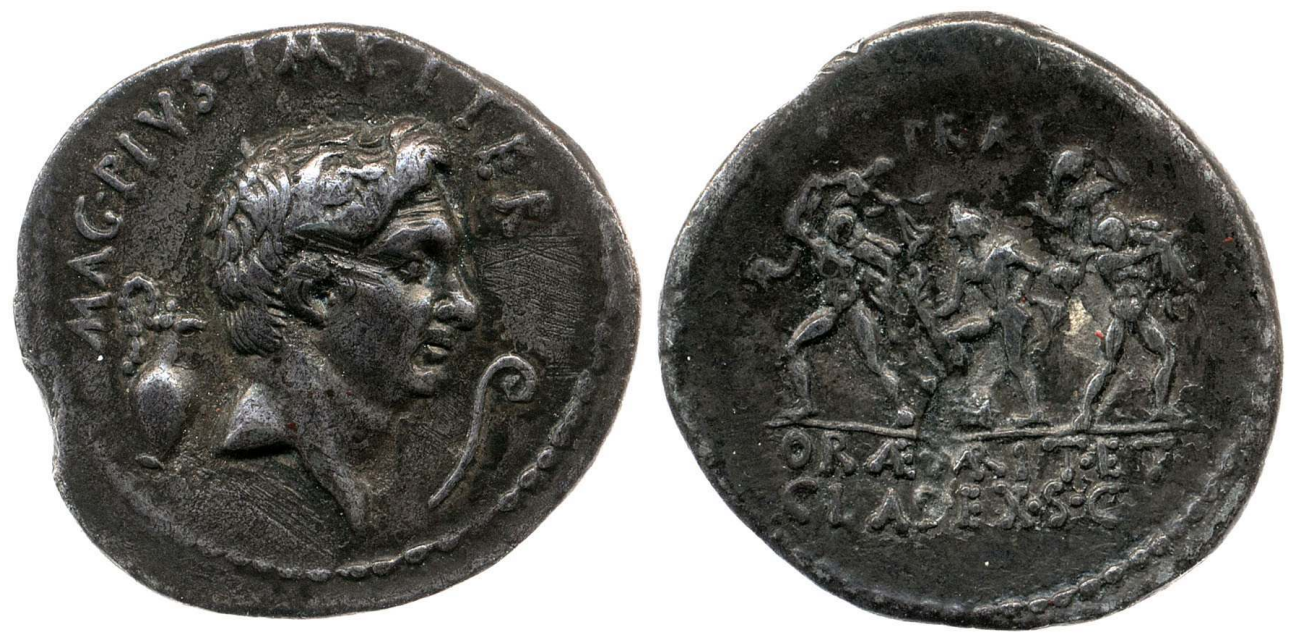

RRC 511/3 B

(C) THE TRUSTEES OF THE BRITISH MUSEUM

14 L'association des symboles de la cruche urceus/gut $(t)$ us et du lituus se réfère donc soit à l'augurat du (pro)magistrat responsable ou bénéficiaire de l'émission soit, dans les cas où ce dernier a un intérêt particulier à mettre en scène sa Pietas, à l'augurat d'un de ses ancêtres. Avant de considérer que l'émission monétaire syllanienne RRC 359/1-2 constitua une exception à ces codes symboliques traditionnels, il convient d'abord de s'assurer à la fois de sa datation et de la date à laquelle Sylla obtint l'augurat.

datation de l'émission par M. H. CRAWFORD (1974, I, p. 80), en 84-83, est désormais établie sur des bases solides. En raison de la faiblesse de certains des arguments avancés par le célèbre numismate, en particulier l'usure des pièces de l'émission RRC 359 plus importante que celle des pièces de l'émission syllanienne $R R C 367^{10}$, cette datation avait soulevé naguère, à juste titre, de sérieuses objections (MARTIN 1989, p. 32-34 ; MACKAY 2000, p. 198-199). L'étude des coins monétaires est venu confirmer, toutefois, une datation haute de l'émission, probablement destinée à financer la reconquête de l'Italie: leur ajustement à 12 heures, qui n'était pas pratiqué à Rome, implique en effet que leur frappe ait été réalisée par un personnel oriental et exclut la possibilité d'une frappe par l'atelier de l'Urbs ${ }^{11}$ (Callatä̈ 1996, p. 30-31 et Hollstein 2000, p. 489, suivis par DRUmmond 2008, p. 393 et ASSENMAKER, à paraitre dans $R N, 2013)$. La question de savoir si la frappe eut lieu en Grèce ou en Asie Mineure avant le débarquement en Italie (HollsteIN 2000, p. 489 ; W OYTEK 2003, p.501, n. 787) ou bien en Italie-même par des ateliers de campagne employant un " personnel qualifié ramené d'Asie Mineure ou de Grèce » (CALlatä̈ 1996, p. $31^{12}$; ASSENMAKER, à paraître dans $R N$, 2013) est secondaire pour notre raisonnement.

16 En revanche, la datation de l'entrée de Sylla dans le collège des augures en 82, traditionnellement retenue depuis E. BADIAN (1968 ; cf. RÜPKE 2005, II, n 1390, p. 926-927 et $\mathrm{n} .4$; ASSENMAKER, à paraitre dans $R N, 2013)$, a été récemment remise en cause par une 
étude d'A. DRUMmOND (2008, en particulier p. 386-401): l'auteur montre de façon convaincante que la disposition législative mentionnée par Dion Cassius, selon laquelle deux personnes apparentées ne pouvaient pas exercer simultanément le même sacerdoce ${ }^{13}$, doit être rattachée à la lex Labiena de 63 et non pas à la lex Domitia de 104, comme le pensait J. NORTH (1990, p. 528-531 ; cf. RÜPKE 2005, III, p. 1636 ; mais, ibidem, II, p. 1271, n. 4). En conséquence, la présence d'un autre Cornelius au sein du collège augural, Lucius Cornelius Scipio Asiagenes (RÜPKE 2005, II, n 1374, p. 922-923), n'empêche aucunement d'envisager que Sylla ait été augure au même moment et de considérer que la prêtrise dont Sylla réclama la restitution en 84 , d'après Appien ${ }^{14}$, ait été l'augurat. En outre, une telle hypothèse a le mérite d'éviter de faire de Sylla une exception en lui attribuant deux sacerdoces, contrairement à la tradition républicaine ${ }^{15}$. Elle permet surtout de faire l'économie d'une interprétation nouvelle de la symbolique augurale présente sur les monnaies, dont on ne voit d'ailleurs pas très bien comment elle aurait pu fonctionner auprès d'une population habituée à y voir non pas une référence aux pouvoirs légitimes du magistrat, mais une référence à l'augurat. Enfin, elle n'empêche nullement de penser, avec R. STEWART (1997, p.179), que de tels symboles aient été mis en avant par des détenteurs d'imperium en manque de légitimité : c'est même ce qui explique, sans doute, l'apparition de ces insignes auguraux sur les monnaies tardo-républicaines, dans un contexte de guerres civiles où les « chercheurs de légitimité ${ }^{16}$ ne manquèrent pas.

L'association de l'urceus/gut(t)us au collège augural n'en demeure pas moins mystérieuse. On sait, toutefois, que les augures, outre le contrôle des auspices des magistrats et les inaugurationes de prêtres et de lieux, accomplissaient certains rites mal connus mais bien attestés, tels l'augurium salutis, l'augurium canarium et les uernisera auguria (P RESCENDI 2005, p. 79). Or au moins certains de ces auguria semblent avoir été associés à un sacrifice :

[On trouvait dans les livres des augures] des instructions pour certains sacrifices qui n'étaient prescrits que rarement aux augures, mais dont il était question pourtant dans le rituel augural; c'est ce qui explique que parmi les insignes des augures figure, à côté du lituus, l'urceus ou cruche ${ }^{17}$ pour les sacrifices.

J. MARQUARDT (1890, p.122) évoque dans ce passage plusieurs sources où il est effectivement question de sacrifices auguraux, voire, plus clairement encore, de sacrifices accomplis par les augures :

Pline, Histoire naturelle, 18, 14: «Voici ce qu'on lit dans les Commentaires des pontifes : "Pour prendre les augures du [sacrifice d'un] chien [Augurio canario agendo], qu'on fixe les dates avant que le blé sorte de sa graine [et] avant qu'il entre dans sa graine" $»^{18}$.

Paul Diacre, 14-15 L. : «Arcanum. Le sens de ce mot vient, soit d'arx, qui est la partie la plus sûre de la ville, soit d'une sorte de sacrifice que les augures font dans la citadelle [sive a genere sacrificii, quod in arce fit ab auguribus], et tellement dérobé à la connaissance du vulgaire, qu'on n'en confie pas même les formules à l'écriture, mais qu'on le célèbre par la mémoire des successeurs; soit du coffre où restent en sûreté les objets qu'on y a renfermés : l'origine du mot arca lui-même vient de arcere $»^{19}$.

Varron, De Lingua latina, 7, 31 : «On dit ambagio comme on dit ambustum, pour "ce qui est brûlé autour", ou comme on dit ambegna, chez les augures, pour la génisse [ ut ambegna bos apud augures] "autour de laquelle on place d'autres victimes" $»^{20}$.

Servius auctus, In Vergilii Aeneidos libros commentarius, 3, 265 : “Dieux, écartez ces menaces": certains tiennent ces paroles pour une catégorie augurale que l'on appelle "invocation". L'invocation est en effet une prière qui est faite pour écarter les malheurs; on accomplit à cet effet ce sacrifice augural [sacrificium augurale] 
après avoir fait monter, comme ici à propos de l'auspice des Harpies, l'invocation

"Dieux, écartez de nous ces menaces", et cetera $»^{21}$. tardif de l'Énéide, en particulier, comme absolument digne de foi. L'auteur même du Commentaire, d'ailleurs, se montre prudent: l'emploi de l'expression "non nulli dictum putant » le prouve. Il en va autrement, en revanche, des deux autres textes. Varron étant très bien renseigné sur les questions augurales ${ }^{24}$, il n'y a en effet aucune raison de douter de la précision qu'il apporte sur la génisse sacrificielle, dont il dit qu'elle se trouve chez les augures (apud augures) : il a trouvé l'information sans doute dans les archives du collège, ou plus vraisemblablement dans un traité sur l'art augural plus facile d'accès, du même type que le Liber auguralis qu'Appius Claudius Pulcher dédia et envoya à Cicéron ${ }^{25}$. Nous ne pouvons certes pas en déduire de façon certaine que les augures accomplissaient eux-mêmes le sacrifice dont les victimes sont ici mentionnées, mais nous ne pouvons pas non plus écarter trop vite cette possibilité : à la différence du passage de Pline l'Ancien, où ce dernier affirme citer le Commentaire des pontifes, Varron, encore une fois, assure avoir trouvé son information "chez les augures ». Quant au témoignage de Paul Diacre, évidemment très tardif, il remonte toutefois, comme on sait, à l'époque augustéenne, en dépit des inévitables altérations et simplifications qui le différencient de la version de Festus et de celle de Verrius Flaccus (GRANDAZZI 1993, p. 64-67). Or ce dernier, un proche d'Auguste, fut particulièrement attentif au vocabulaire augural, ses lemmes " auguraux » ou "auspiciaux» reposant sur des travaux d'érudits républicains ou augustéens spécialistes du droit augural (GRANDAZZI 1993, p. 266). La crédibilité de ces témoignages littéraires sur les sacrifices accomplis par les augures est renforcée par le témoignage épigraphique des Fastes de Préneste, où l'on peut lire, à la date du 17 janvier : pontifices, a [ugures, XVviri s(acris) f(aciundis), VII]vir(i) epulonum victumas in/m[ola]nt (D EGRASSI 1963, p. 114-115 et p. 401).

Dans ces conditions, il est tentant d'expliquer l'association de l'urceus/gut(t)us au lituus et au collège des augures par ces mystérieux "sacrifices auguraux ». Il ne s'agit toutefois que d'une hypothèse, que nous avançons, après d'autres (voir MARQUARDT 1890, p. 122 ; RE , WissowA 1896, s.v. augures, col. 2329-2330 ; DRUMMOND 2008, p. 391, n. 111), avec une très grande prudence ${ }^{26}$. 


\section{BIBLIOGRAPHIE}

ALFÖLDI A. (1956), « The main aspects of political propaganda on the coinage of the Roman republic ", dans Carson R. A. G. et Sutherland C. H. V. éd., Essays in Roman coinage presented to H. Mattingly, Oxford, p. 63-95.

Allély A. (2012), La déclaration d'hostis sous la République romaine, Scripta Antiqua 47, Bordeaux. ASSENMAKER P. (sous presse), De la victoire au pouvoir. Développement et manifestations de l'idéologie impératoriale à l'époque de Marius et Sylla.

ASSENMAKER P. (à paraître dans RN, 2013), « L. Sulla imperator et imperator iterum : pour une réévaluation de la chronologie des émissions monétaires de Sylla (RRC 367-368 et 359)».

BADIAN E. (1968), « Sulla's Augurate », Arethusa 1.1, p. 26-46.

BAYET J. (1950), « Les Feriae Sementivae et les Indigitations dans le culte de Cérès et de Tellus », RHR 137, p. 172-206.

CALLATAŸ F. DE (1996), Les monnaies grecques et l'orientation des axes, Milan.

CARson R. A. G. et Sutherland C. H. V. (1984), The Roman Imperial Coinage (abrégé RIC), I, Londres.

Catalano P. (1960), Contributi allo studio del diritto augurale, I, Turin.

CRAWfORD M. H. (1974), Roman Republican Coinage (abrégé RRC), I et II, Cambridge.

Daremberg et SAglio = Daremberg C., SAglio E., Pottier E. et LAfAye G. éd., Dictionnaire des Antiquités grecques et romaines, Paris, 1877-1919.

DAREMberg et SAglio, Grenier A. (1911), IV.2, s.v. situla, p. 1357-1360.

DAREMBERG et SAGLio, KARo G. (1907), IV.1, s.v. praefericulum, p. 622.

DAREMBerg et SAGLIO, PotTiER E. (1896), II.2, s.v. guttus, p. 1674.

DAREmberg et SAglio, PotTier E. (1911), IV.2, s.v. simpulum, p. 1345-1346.

DAREMBerg et SAglio, Pottier E. (1919), V, s.v. urceus, p. 604.

DAREMBerg et SAglio, SAglio E. (1887a), I.2, s.v. capis, p. 896.

DAREMBerg et SAGLio, SAglio E. (1887b), I.2, s.v. culullus, p. 1587.

DEgRASSI A. (1963), Inscriptiones Italiae. XIII.2. Fasti Anni Numani et Iuliani, Rome.

DeLATTE L. (1937), « Recherches sur quelques fêtes mobiles du calendrier romain. IV. Augurium canarium ", AC 6, 1, p. 93-102.

DRummond A. (2008), « The Ban on Gentiles Holding the Same Priesthood and Sulla's Augurate », Historia 57, 4, p. 367-407.

Estiot S. (2006), " Sex. Pompée, la Sicile et la monnaie. Problèmes de datation », dans Champeaux J. et Chassignet M. dir., Aere perennius. Hommage à Hubert Zehnacker, Paris, p. 125-153.

FEARS J. R. (1975), « The Coinage of Q. Cornificius and Augural Symbolism on Late Republican Denarii », Historia 24, p. 592-602. 
FLESS F. (1995), Opferdiener und Kultmusiker auf stadtrömischen historischen Reliefs : Untersuchungen zur Ikonographie, Funktion und Benennung, Munich.

FLOBERT P. (1985), Varron. La langue latine. Live VI, Paris.

FRIER B. W. (1967), « Augural Symbolism in Sulla's Invasion of 83 », ANSMusN 13, p. 111-118.

FRIER B. W. (1969), « Sulla's Priesthood », Arethusa 2, p. 187-199.

GRANDAZZI A. (1993), « “Intermortua iam et sepulta uerba” (Festus, 242 L) : les mots de la divination chez Verrius Flaccus. I et II. », RPh 67, p. 57-73 et 263-285.

GRUEBER H. A. (1910), Coins of the Roman Republic in the British Museum, II, Londres (réimpression 1970).

HANo M. (1986), « Les Autels des Lares Augusti », ANRW II, 16, 3, Berlin-New York, p. 2333-2381.

HILGERS W. (1969), Lateinische Gefässnamen : Bezeichnungen, Funktion und Form römischer Gefässe nach den antiken Schriftquellen, Düsseldorf.

HÖLSCHER T. et SCHÖRNER G. (2005), « III. Libation. A. Römisch », dans ThesCRA, V.2.b, Los Angeles, p. 204-212.

HOLLSTEIN W. (1993), Die stadtrömische Münzprägung der Jahre 78-50 v. Chr. zwischen politischer Aktualität und Familienthematik: Kommentar und Bibliographie, Quellen und Forschungen zur antiken Welt 14, Munich.

HoLLSTEIN W. (2000), « Die Stempelstellung : ein ungenutztes Interpretationskriterium für die Münzprägung der römischen Republik », dans Kluge B. et Weisser B. éd., XII. Internationaler Numismatischer Kongress Berlin 1997, Berlin, p. 487-491.

KeAveney A. (1982), « Sulla Augur : Coins and Curiate Law », AJAH 7, p. 150-171.

LACAM J.-C. (2008), « Le sacrifice du chien dans les communautés grecques, étrusques, italiques et romaines. Approche comparatiste », MEFRA 120, 1, p. 29-80.

LAIGNOUX R. (à paraître), « La construction du pouvoir personnel durant les années 44-29 : processus de légitimation », manuscrit de la thèse de doctorat soutenue à Paris 1 PanthéonSorbonne en 2010.

LE BONNIEC H. (1958), Le Culte de Cérès à Rome, des origines à la fin de la République, Paris.

Le BonNiec H. avec la collaboration de Le Boeuffle A. (1972), Pline l'Ancien. Histoire Naturelle. Livre XVIII, Paris.

LINDERSKI J. (1986), « The Augural Law », ANRW II, 16, 3, Berlin-New York, p. 2146-2312.

LINDERSKI J. (1996), « Q. Scipio Imperator », dans idem éd., « Imperium sine fine » :

T. Robert S. Broughton and the Roman Republic, Historia-Einzelschriften 105, Stuttgart.

LUCE T. J. (1968), « Political Propaganda on Roman Republican Coins : circa 92-82 B.C. », AJA 72, 1, p. 25-39.

MACKAY C. S. (2000), « Sulla and the Monuments : Studies in His Public Persona », Historia 49, 2, p. 161-210.

MARQUARDT J. (1890), Le culte chez les Romains, II, Paris, trad. fr. J-B. Brissaud de la $2^{\mathrm{e}}$ édition allemande du volume III [1885²] de Die römische Staatsverwaltung (Manuel des Antiquités romaines, XII-XIII) 
MARTIN T. R. (1989), « Sulla Imperator Iterum, the Samnites and Roman Republican Coin Propaganda », SNR 68, p. 19-44.

MORAWIECKI L. (1996), « Pontificalia atque auguralia insignia and the Political Propaganda in the Coinage of the Roman Republic », Notae Numismaticae 1, p. 37-57.

NoRTH J. (1990), « Family strategy and priesthood in the Late Republic », dans Andreau J. et Bruhns H. éd., Parenté et stratégies familiales dans l'antiquité romaine, collection de l'École française de Rome 129, Rome, p. 527-543.

PERA R. (1976), « Venere sulle monete da Silla ad Augusto. Aspetti storico-politici », dans Contributi di storia antica in onore di Albino Garzetti, Genève, p. 241-268.

PRESCENDi F. (2005), « Augures publici populi Romani Quiritium », dans ThesCRA, V.2.a, Los Angeles, p. 77-80.

Prosdocimi A. (1985-1990), «Augurium canarium. Appendici : Vernisera e altri auguri agrari. Ceres e Cerialia. Sementivae e Feriae delle semine ", Abruzzo 23-28, p. 323-340.

RE = WIssowA G. et alii éd., Paulys Realencyclopädie der classischen Altertumswissenschaft, StuttgartWeimar, 1894-1980.

RE, LEONARD (1927), III.A.1, s.v. simpulum, col. 213-216.

RE, LEONARD (1927), III.A.1, s.v. situlus, col. 415-417.

RE, SAMTER (1901), IV.2, s.v. culullus, col. 1753-1754.

RE, WISSOWA (1896), II.2, s.v. augures, col. 2313-2344.

RE, ZAHN (1912), VII.2, s.v. guttus, col. 1953.

Rivero-Gracia M. P. (2006), « Muros de aire : auspicia, imperium y delimitación del espacio sagrado romano en tierras bárbaras ", dans Bedon R., Liébert Y. et Mavéraud H. éd., Les espaces clos dans l'urbanisme et dans l'architecture en Gaule romaine et dans les régions voisines. Hommage à Raymond Chevallier. Colloque international tenu à la Faculté des lettres et des sciences humaines de Limoges, 11 et 12 juin 2004, p. 397-406.

RÜPKE J. et GLOCK A. (2005), “Fasti sacerdotum” die Mitglieder der Priesterschaften und das sakrale Funktionspersonal römischer, griechischer, orientalischer und jüdisch-christlicher Kulte in der Stadt Rom von 300 v. Chr. bis 499 n. Chr., II et III, PAwB 12, 2, Stuttgart.

SAVAgner M. A. (1846), Sextus Pompeius Festus, Paris.

SCHEID J. (1981), « Le délit religieux dans la Rome tardo-républicaine », dans Le délit religieux dans la cité antique (table ronde, Rome, 6-7 avril 1978), collection de l'École française de Rome 48, Rome, p. 117-171.

SIEBERT A. V. (1999), Instrumenta sacra. Untersuchungen zu römischen Opfer-, Kult- und Priestergeräten, Berlin-New York.

STEWART R. (1997), « The Jug and Lituus on Roman Republican Coin Types : Ritual Symbols and Political Power ", Phoenix 51, 2, p. 170-189.

SzemLeR G. J. (1974), « The Dual Priests of the Republic », RhM 117, p. 72-86.

TAYLOR L.-R. (1944), «Symbols of the augurate on coins of the Caecilii Metelli », AJA 48, 4, p. 352-356. 
TAYLOR L.-R. (1966), Roman Voting Assemblies from the Hannibalic War to the Dictatorship of Caesar, Ann Arbor.

URLICHS K. L. (1857), Chrestomathia Pliniana, Berlin.

VAleton I. M. J. (1889), « De modis auspicandi Romanorum », Mnemosyne n.s. 17, 4, p. 418-452.

VAN HAeperen F. (2002), Le Collège pontifical ( $3^{e}$ s. a. C.-4 $4^{e}$ s. p. C.). Contribution à l'étude la religion publique romaine, Bruxelles-Rome.

VON SCHAEWEN R. (1940), Römische Opfergeräte, ihre Verwendung im Kultus und in der Kunst, Berlin.

Wissowa G. (1912² [1902]), Religion und Kultus der Römer, Handbuch der klassischen

Altertumswissenschaft, 5, 4, Munich.

WоYтек B. (2003), « Arma et Nummi ». Forschungen zur römischen Finanzgeschichte und Münzprägung der Jahre 49 bis 42 v. Chr., Vienne.

\section{NOTES}

1. Voir, cependant, Cicéron, Epistulae ad Atticum, 9, 9, 3.

2. Toutes les dates de l'article s'entendent avant J.-C.

3. Pour les cruches du type urceus-gut $(t)$ us, voir DAREMBERG et SAGLIO, PotTIER 1919, s.v. urceus, p. 604 et DAREMBerg et SAglio, PotTier 1896, s.v.guttus, p.1674; RE, ZAHN 1912, s.v.guttus, col. 1953 ; TAYLOR 1944, p. 353 ; SIEBERT 1999, p. 133, 221-222 et 240-241 (avec les remarques critiques d'A. DRUMmond 2008, p. 392, n. 112, sur la typologie de d'A. V. SIEBERT). Cf. VON SCHAEWEN 1940, p. 15-23; HILGERS 1969, p. 58-59; FlesS 1995, p. 17-19. Cette cruche à anse a été souvent interprétée comme une capis (ALFÖLDI 1956, p. 86 ; GRUEBER 1910, par exemple p. 460, n. 1 (suite) ou p. 560 ; PERA 1976, p. 244 ; MORAWIECKI 1996, p. 45 ; ESTIOT 2006, p. 128 ; A SSENMAKER, sous presse), vase qui semble pourtant devoir être associé au collège pontifical (DAREMBERG et SAGLIO, $\mathrm{S}$ AGLIO 1887a, s.v.capis, p. 896 ; TAYLOR 1944, p. 353 ; LINDERSKI 1996, p. 175-176; SIEBERT 1999, p. 211-212. Cf. Tite-Live, Histoire romaine, 10, 7, 10, où capis renvoie aux pontifes et lituus aux augures). Les désignations de cette cruche comme un simpuvium/simpulum (HöLSCHER et SCHÖRNER 2005, p. 206-207) ou comme une situla/sitella (FRIER 1967, p. 117 ; LUCE 1968, p. 27 ; HOLLSTEIN 1993, p. 285 ; Rivero-Gracia 2006, p. 147-148) doivent elles aussi être rejetées: comme la capis, le simpuvium/simpulum semble être lié collège pontifical (DAREMBERG et SAGLIO, PotTIER 1911, s.v. simpulum, p. 1345-1346 ; RE, LEONARD 1927, s.v. simpulum, col. 213-216 ; LINDERSKI 1996, p. 176 ; S IEBERT 1999, p. 132-133, 236-239) ; la situla/sitella n'est pas attestée comme instrument de culte par les sources littéraires (DAREMBERg et SAGLio, GRENIER 1911, s.v. situla, p. 1357-1360 ; RE, LeonARD 1927, s.v. situlus, col.415-417; Siebert 1999, p.60. G.Karo (D AREMBERg et SAGLiO, Karo 1907, s.v. praefericulum, p. 622), M. HANO 1986, p. 2366 et, plus récemment, A. V. S IEBERT 1999, p. 56 rejettent également l'emploi du terme praefericulum pour désigner une cruche.

4. Cicéron, De Republica, 2, 26 ; De Haruspicum responso, 14 et 18; De Natura deorum, 1, 122 et 3, 5.

5. Pour le simpuvium/simpulum, voir n. 3. Pour le culullus, voir DAREMBERG et SAGLio, SAGLio 1887b, s.v. culullus, p.1587; RE, SAMTER 1901, s.v. culullus, col.1753-1754; SIEBERT 1999, p.132-133, 216-217.

6. SZEMLER 1974, p. 73-78.

7. Voir la note précédente.

8. Le thème de la Pietas était déjà présent sur l'émission d'Espagne RRC 477 : cf. E STIOT 2006, p. 132-133.

9. ESTIOT 2006, p. 140. Cf. RÜPKE 2005, n² 2758, p. 1219. 
10. M. H. CRAWford (1974, I, p. 80, n. 3) est d'ailleurs le premier à reconnaître la faiblesse de l'argument : «I am aware that the evidence of a single small hoard is not decisive ».

11. S'il est certes possible d'envisager que l'émission ait été frappée à l'atelier de Rome, après la victoire syllanienne, par le personnel ramené d'Orient, l'absence d'ajustement des coins de l'émission 376 indique, comme me l'a signifié $P$. Assenmaker, que Sylla reprit très vite le contrôle de l'atelier de l'Urbs et des équipes d'artisans qui y étaient attachées.

12. P. Assenmaker m'informe que F. de Calataÿ a aujourd'hui rallié la position plus traditionnelle de W. Hollstein et de B. Woytek.

13. Dion Cassius, Histoire romaine, 39, 17, 1-2.

14. Appien, Guerres civiles, 1, 79, 362. A. KeAVenay (1982, p. 151) a souligné combien il était difficile de concevoir qu'un hostis, qui perdait jusqu'à sa citoyenneté (cf. Cicéron, Catilinaires, 4, 10), ait pu conserver l'augurat. Sur la déclaration d'hostis sous la République romaine, voir désormais ALLÉLY 2012.

15. Voir, ci-dessus, n. 6. E. BADIAN (1968, p. 39-40) pensait au pontificat et J. RÜPKE (2005, nº 1390, p. 927, n. 1) au décemvirat sacris faciundis.

16. Pour l'expression "chercheurs de légitimité », voir R. LAIGNoux (à paraître), qui l'emploie pour les prétendants au pouvoir de la période triumvirale.

17. Nous avons corrigé là une erreur de traduction de J-B. Brissaud, qui avait rendu «die Opferkanne (urceus) » de l'édition originale par «l'urceus ou bâton pour les sacrifices ».

18. Pline, Histoire naturelle, 18, 14 : Ita enim est in commentariis pontificum: Augurio canario agendo dies constituantur, priusquam frumenta vaginis exeant et antequam in vaginas perueniant. Trad. VAN H AEPEREN 2002, p. 270-271, d'après trad. LE BONNIEC 1972, pour la CUF. La correction du «et » (et antequam in vaginas...) en "nec", proposée par K. L. URLICHS 1857, p. 224, et souvent suivie, en particulier par L. DelatTE 1937, p. 93, n'est pas heureuse : voir, notamment, les commentaires de J. BAYet 1950, p. 186, n. 2 et de H. Le BonNiEC 1958, p. 124, n. 3. Pour une autre proposition de correction, voir PRosDocimi 1985-1990, p. 325-326. Sur les sacrifices de chiens de manière plus générale, voir LACAM 2008.

19. Paul Diacre, 14-15 L. : 'Arcani' sermonis significatio trahitur siue ab arce, quae tutissima pars est urbis; siue a genere sacrificii, quod in arce fit ab auguribus, adeo remotum a notitia vulgari, ut ne litteris quidem mandetur, sed per memoriam successorum celebretur; siue ab arca, in qua quae clausa sunt, tuto manent, cuius ipsius origo ab arcendo pendet. Trad. SAVAGNER 1846.

20. Varron, De Lingua latina, 7, 31 : Ambagio dicta ut ambustum, quod circum ustum est, ut ambegna bos apud augures, quam circum aliae hostiae constituuntur. Trad. pers.

21. Servius auctus, In Vergilii Aeneidos libros commentarius, 3, 265: 'Dii prohibete minas' hoc per speciem auguralem quae inuocatio appellatur non nulli dictum putant. Inuocatio autem est precatio, uti auertantur mala, cuius rei causa id sacrificium augurale peragitur, ut hic de auspicio harpyiarum inuocatione suscepta «dii prohibete minas » et cetera. Trad. pers. Cf. Servius auctus, In Vergilii Aeneidos libros commentarius, 12, 176 : Speciem augurii, quae precatio maxima appellatur. Voir DELATTE 1937, p. 101-102 : «l'augurium dont il est question ici ne comporte pas une observation du ciel ou du vol des oiseaux, les deux objets de la divination pratiquée à l'occasion de l'auguration, mais un sacrifice dit augural et une invocation déprécatoire. »

22. Wissowa $1912^{2}$ [1902], p. 524 : « Mit den caerimoniae et sacra, auf welche sich die Tätigkeit der Pontifices erstreckt, haben die Augurn nichts $\mathrm{zu}$ tun [...] ist mit dem augurium eine Opferhandlung verbunden, so fällt ihre Ausführung den Pontifices zu, im Augurencollegium gibt es keinen Flamen.»; CATALANo 1960, p. 341 : «E' certo errore grave, e gravido di conseguenze, confondere con l'augurium le distinte azioni religiose della preghiera e del sacrificio. » 23. LINDERSKI 1986, p. 2254, n. 421 ; cf. p. 2222-2223 ; idem, 1996, p. 176 et n. 120. Cf. CATALANO 1960, p. 356: «Si può anche ammettere che le azioni religiose dette nel loro complesso auguria implicassero preghiere o sacrifici da parte degli auguri, ma bisogna sempre distinguere: la 
preghiera iniziale, anteriore all'inaugurazione; l'inaugurazione (augurium in senso stretto, consultazione della volontà divina); la preghiera o il sacrificio che erano oggetto dell'inaugurazione. "

24. Le prouvent, entre autres, les développements de Varron sur la typologie augurale des genera agrorum (De Lingua latina, 5, 33) et sur les templa (De Lingua latina, 7, 8-10), sur le pomerium (De Lingua latina, 5, 143), sur la prise d'auspices précédant toute réunion du Sénat (Varron in AuluGelle, Noctes Atticae, 14, 7, 9), sur les auspices du questeur (De Lingua latina, 6, 91) et du censeur (De Lingua latina, 6, 86-87) et les extraits qu'il cite des Libri Augurales, à cinq reprises rien qu'au livre 6 du De Lingua latina: voir l'introduction de P. FLOBERT 1985 à son édition du livre 6, pour la CUF, p. XIV.

25. Cicéron, Epistulae ad Familiares, 3, 4, 1 (=67 Shackleton Bailey) ; Festus, s.v. sollistimum, p. 386 L. (Liber auguralis disciplinae).

26. La circonspection est d'autant plus de mise qu'une hypothèse alternative a été proposée naguère par L.-R. TAYLOR 1966, p. 74 ; suivie par LINDERSKI 1996, p. 176-177. Selon elle, l'urceus renverrait au tirage au sort des provinces des consuls et des préteurs (la sortitio), qui était contrôlé par le collège augural et devait avoir lieu dans un templum inauguré (LINDERSKI 1986, p. 2173-2175 et p. 2193-2194, n. 173 ; STEWART 1998, p. 12-51). Nous noterons cependant que, dans l'un des rares passages où il est question de l'aspect augural de la sortitio (Tite-Live, Histoire romaine, 41, 18, 8), il est fait mention de sitella et non pas d'urceus: Valerium auspicato sortitum constabat, quod in templo fuisset; in Petilio id uitii factum postea augures responderunt, quod extra templum sortem in sitellam <...> in templum latam foris ipse <...> oporteret. Le signe <...> indique une lacune dans le manuscrit. Voir aussi les réticences d'A. DRUMMOND 2008, p. 391, n. 111.

\section{RÉSUMÉS}

Cette étude s'inscrit en faux contre la tendance actuelle à considérer que sylla modifia la signification traditionnelle du bâton augural (le lituus) sur les émissions monétaires. En l'associant à une cruche sacrificielle - fréquemment interprétée comme pontificale -, le célèbre imperator aurait voulu symboliser non pas son appartenance au collège augural - qu'il n'aurait intégré que plus tard -, mais la double légitimité de son imperium : légitimité auspiciale de la loi curiate et des auspices d'investiture (le lituus), légitimité des sacrifices d'entrée en charge (la cruche). En réalité, cette cruche n'est jamais associée, sur les monnaies, ni aux instruments pontificaux ni à un pontife (à l'exception de César et d'Auguste, à la fois augures et pontifes). Inversement, elle est toujours associée aux instruments auguraux ou à un augure (à l'exception de [pro]magistrats souhaitant souligner leur pietas envers un ancêtre augure). Cette cruche renvoie peut-être à des cérémonies augurales mal connues. Il est plus convaincant de changer la date d'entrée de Sylla au sein du collège des augures, que de modifier l'interprétation traditionnelle des symboles monétaires auguraux.

This study strongly denies the current trend to consider that Sylla changed the traditional meaning of the augural stick (lituus) on monetary emissions. In combination with a sacrificial jug - frequently interpreted as pontifical -, the famous imperator would have liked to symbolize not his belonging to the augural college - that he would have joined later -, but the dual legitimacy of his imperium: auspicial legitimacy of the curiate law and of auspices of investiture (the lituus), legitimacy of sacrifices of investiture (the jug). In reality, this jug is never associated, on coins, 
neither to pontifical instruments nor to a pontiff (except for Caesar and Augustus, both augurs and pontiffs). On the other hand, it is always associated with augural instruments or an augur (except for [pro]magistrates wishing to emphasise their pietas towards an ancestor augur). This jug refers perhaps to little known augural ceremonies. It's more convincing to change the date of Sylla's entrance to the augural college, than modify the traditional interpretation of augural symbols on coins.

INDEX

Mots-clés : République romaine, monnaie, Sylla, augurat, lituus, cruche, auspices, sacrifice, imperium

Keywords : Roman Republic, coins, Sylla, augurate, lituus, jug, auspices, sacrifice, imperium

\section{AUTEUR}

YANN BERTHELET

ANHIMA - UMR 8210 